\title{
Transesophageal echocardiography training: looking forward to the next step
}

\author{
André Y. Denault, MD • Antoine G. Rochon, MD
}

Received: 16 September 2010/Accepted: 14 October 2010/Published online: 13 November 2010

(C) Canadian Anesthesiologists' Society 2010

In this issue of the Journal, Jerath et al. ${ }^{1}$ describe how an interactive online transesophageal echocardiography (TEE) teaching module with a pretest/posttest design significantly improved the performance of multidisciplinary trainees. Why is such an initiative so important in 2011? Since the use of an esophageal echocardiographic probe was first described in $1976^{2}$ there have been significant advances in both TEE and transthoracic echocardiography (TTE) in terms of equipment, indications, training requirements, certification, and continuous medical education. As echocardiography (TEE and, ultimately, goal-oriented TTE) becomes an integral part of the training not only of cardiac anesthesiologists but also of general anesthesiologists and critical care physicians, some issues need to be addressed.

\section{Indications for the use of TEE}

As described by Frazin et al., ${ }^{2}$ TEE was used originally in patients with chronic obstructive lung disease due to suboptimal TTE image acquisition because of alterations of the thoracic cavity. Similarly in the operating room (OR),

A. Y. Denault, MD $(\bowtie) \cdot$ A. G. Rochon, MD

Department of Anesthesiology, Montreal Heart Institute and University de Montréal, 5000 Belanger Street, Montreal, QC H1T 1C8, Canada

e-mail: andre.denault@umontreal.ca

A. Y. Denault, MD

Centre Hospitalier de l'Université de Montréal (CHUM),

Montreal, QC, Canada access to the chest is often limited due to sterility issues or for technical reasons. Consequently, transesophageal echocardiography became the instrument of choice for monitoring and diagnosing various causes of hemodynamic instability in the OR, particularly during cardiac surgery. The original 1996 guidelines for the use of TEE were recently revised. ${ }^{3}$ The guidelines were updated in 2010 by an American Society of Anesthesiologists (ASA)/Society of Cardiovascular Anesthesiologists Task Force ${ }^{4}$ composed of nine anesthesiologists from private and academic practices and from various geographic areas of the United States. The Task Force included also two cardiologists, one representing the American College of Cardiology and the other representing the American Society of Echocardiography, as well as two consulting methodologists from the American Society of Anesthesiologists Committee on Standards and Practice Parameters. Their literature review covered a 16-year period from 1994 through 2009, and a total of 457 articles were identified out of the 8,000 citations initially considered. From this analysis, guidelines were consensually written. For instance, an agreement was reached that TEE should be used in all thoracic aortic and cardiac procedures. In addition, TEE should be used, if available, in cases where unexplained life-threatening circulatory instability persists despite appropriate therapy for non-cardiac surgery. Such cases include the following: unexplained persistent hypotension or hypoxemia, anticipated life-threatening hypotension, lung or liver transplantation, major thoracic or abdominal trauma, and open abdominal aortic procedures. Further, TEE should also be used in the critical care setting in cases where persistent hypotension or hypoxemia remains unexplained. These recommendations should lead to a significant increase in the demand for TEE training. In this respect, the work of Jerath et al. ${ }^{1}$ is indeed timely. 


\section{Who should be trained in TEE?}

Transesophageal echocardiography should be used only by individuals who are adequately trained. Increasingly, cardiac anesthesiologists must be certified in perioperative TEE by organizations, such as the American National Board of Echocardiography (NBE) (www.echoboard.com), in order to obtain hospital privileges in echocardiography. Training curricula include cardiac anesthesia fellowship programs and intensive programs for anesthesiologists already in practice. Prior to 2010, only advanced certification was available through the NBE. Today a new level of certification is available called Basic Perioperative Transesophageal Echocardiography. This certification is oriented toward the basic knowledge that non-cardiac anesthesiologists and critical care physicians should master in order to use TEE. The objectives of the basic and advanced NBE examinations are listed in Tables 1 and 2.

\section{What precautions should be taken before using TEE?}

At the Montreal Heart Institute, TEE examination is included in the general consent form. Unless contraindicated, we routinely perform a complete cardiac TEE study following induction of anesthesia and endotracheal intubation. The TEE probe is kept in place until the end of the procedure. It is particularly important to insert the TEE probe before the administration of heparin for cardiopulmonary bypass (CPB), since inserting the probe during CPB has been associated with gastrointestinal bleeding. ${ }^{5}$ Contraindications to TEE include esophageal or gastric pathologies that would predispose to perforation, respiratory distress in the non-intubated patient, cervical spine instability, and severe coagulopathy. With the increase of

Table 1 National Board of Echocardiography exams and certifications

Basic PTE examination objectives

1. Patient safety considerations

2. Echocardiographic imaging: acquisition and optimization

3. Normal cardiac anatomy and imaging plane correlation

4. Global ventricular function

5. Regional ventricular systolic function and recognition of pathology

6. Basic recognition of cardiac valve abnormalities

7. Identification of intracardiac masses in non-cardiac surgery

8 Basic perioperative hemodynamic assessment

9. Related diagnostic modalities

10. Basic recognition of congenital heart disease in the adult

11. Surface ultrasound for vascular access

PTE Perioperative transesophageal echocardiography
Table 2 National Board of Echocardiography exams and certifications

Advanced PTE examination objectives

1. Principles of ultrasound

2. Transducers

3. Imaging

4. Principles of Doppler ultrasound

5. Doppler flow profiles for normal and abnormal physiology

6. Quantitative echocardiography

7. Artifacts and pitfalls of imaging

8. Equipment, infection control, and safety

9. Normal anatomy and flow during the complete examination

10. Myocardial ischemia and segmental ventricular function

11. Ventricular function and hemodynamics

12. Recognizing intracavitary contents

13. Echocardiographic manifestations of congenital heart disease in adult patients

14. Hypertrophic obstructive cardiomyopathy

15. Dilated and restricted cardiomyopathies

16. Echocardiography for the pericardium and extracardiac anatomy

17. Echocardiography during cardiac surgery

18. Assessing heart valves during the perioperative period

19. Indications for transesophageal echocardiography

20. Non-TEE imaging and other diagnostic modalities

PTE Perioperative transesophageal echocardiography; TEE transesophageal echocardiography (from www.echoboard.com)

routine use of TEE outside the cardiac OR, it is important that questions related to esophageal pathologies be added to the routine preoperative evaluation form. Despite these precautions, esophageal perforation remains a risk of perioperative TEE. In our institution, three such perforations occurred within the previous 15,000 examinations since 1999 (information obtained from the Montreal Heart Institute TEE database). For this reason, other alternatives should be made available to the anesthesiologist in cases of high-risk patients.

\section{Alternatives to TEE}

In the cardiac OR, epicardial echocardiography can be used as a substitute for TEE. Guidelines for performing an epicardial examination have been published. ${ }^{6}$ In the noncardiac setting or in the recovery room, there have been significant developments in bedside echocardiography for hemodynamically unstable patients following tracheal extubation and for patients with contraindications to TEE. An approach combining both physical examination and bedside echocardiography has improved the clinical diagnosis and management of acutely ill patients. Such 
point-of-care echocardiographic examinations are usually "directed" or "focused" toward a specific clinical question and are significantly shorter in duration (less than six minutes in some studies) than conventional echocardiography. ${ }^{7,8}$ The goal of such an exam is not to perform a complete and comprehensive study but rather to enhance and extend the physical examination.

Transthoracic echocardiography is non-invasive and more readily available than TEE; therefore, it should be the modality of choice for performing a goal-directed echocardiographic examination. ${ }^{9}$ So far, it has not been used widely in the intensive care unit and the OR, because only a few intensivists and anesthesiologists are TTE-trained. ${ }^{10}$ Nonetheless, over the past few years, bedside echocardiography has been performed increasingly by noncardiologists, intensivists, and anesthesiologists to provide diagnostic information that cannot be assessed by physical examination alone. ${ }^{11-13}$ The TTE exam, called FOCUS (FOcused Cardiac Ultrasound Study) or FOCCUS (FOcused Critical Care Ultrasound Study), is defined as a TTE performed with specific limited objectives. ${ }^{10}$ The FOCUS objectives include the assessment of both left and right ventricular function, the assessment of the pericardial space for effusion and tamponade, and the assessment of volume status. Additional FOCCUS objectives include the assessment of lung pathology, abdominal fluid, vessels, etc. Eventually, TTE competency will have to be established following predetermined levels of training (e.g., levels 1 to 4). Some training guidelines have been proposed already, ${ }^{14-16}$ including a consensus between the American College of Chest Physicians and the Société de Réanimation de Langue Française. ${ }^{17}$

\section{Standard and advanced approaches to learning echocardiography}

Learning echocardiography includes directed readings and lectures, supervised intraoperative or intensive care unit experiences, and echocardiography fellowships. In this issue of the Journal, Jerath et al. ${ }^{1}$ present their experience in developing an online program that allows users to visualize each of the 20 standard diagnostic views of TEE in conjunction with a three-dimensional (3D) heart model. In order to validate the educational value of this teaching material, the authors analyzed how trainees performed after having access to the educational material for an average of $130 \mathrm{~min}$. They observed a significant increase of $31 \%$ in their test scores. The trainees also found this program to be significantly useful, giving it a score of 4.7 out of 5 . In
Figure Correlation between transesophageal mid-esophageal long-axis view $(\mathbf{A}, \mathbf{B})$ and transthoracic parasternal longaxis view $(\mathbf{C}, \mathbf{D})$. The two views will provide the same information

Figure Corrélation entre la coupe transœsophagienne mid-œsophagienne en axe long $(\mathbf{A}, \mathbf{B})$ et la coupe transthoracique parasternale en axe long (C, D). Les deux coupes fourniront les mêmes informations
A

The first step

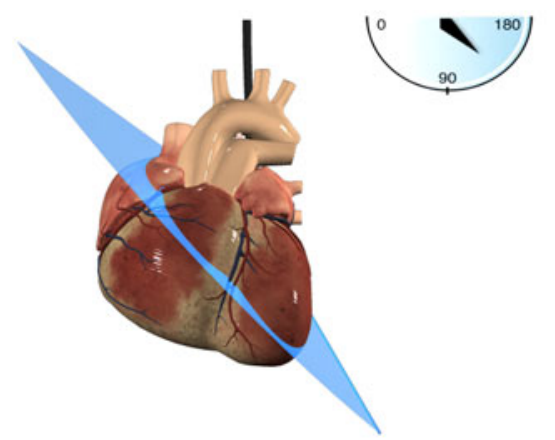

B

Transesophageal view: Mid-esophageal long-axis view

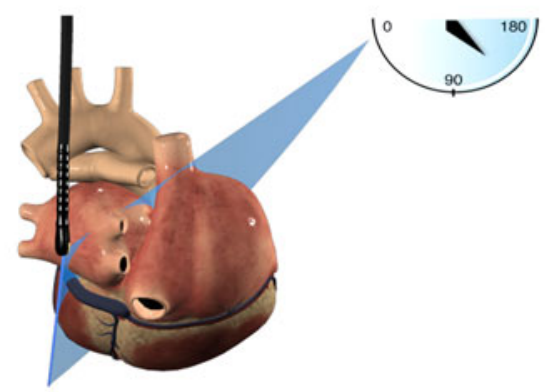

C

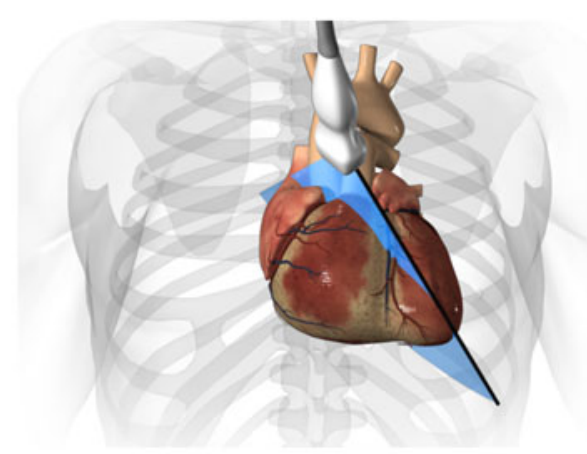

D

Transesophageal view: Parasternal long-axis view

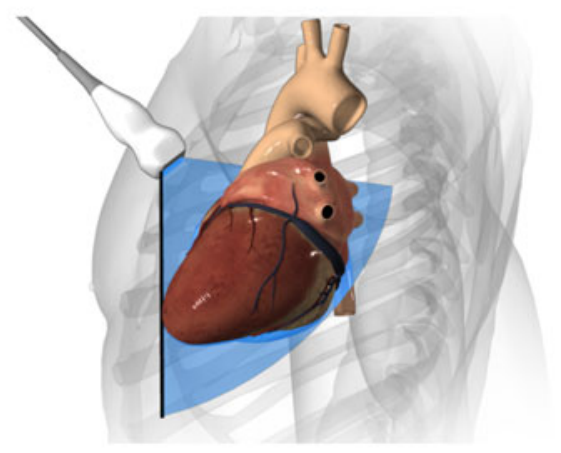


addition, Jerath et al. ${ }^{1}$ demonstrated that the test made it possible to distinguish between novice and expert users of TEE. This website is highly recommended for individuals training in TEE and represents a significant contribution to education by Dr. Jerath and the Toronto group of anesthesiologists. Furthermore, such initiatives open the way for recognizing pathologies and for learning how to use TTE. In fact, TTE images simply offer a different perspective of the TEE images (Figure). By extension, this initiative could be adapted for teaching ultrasound for the chest, the abdomen, and other great vessels. In addition, such educational tools would elegantly complement the use of simulators. The major advantage of simulation is that the echocardiography student can be exposed to various rare pathologies, such as aortic dissection and cardiac tamponade. There is a definitive need for added resources to teach the skill. The web site (http://pie.med.utoronto.ca/ TEE/index.htm) now offers a validated teaching tool that is freely accessible to all. Ultimately, training should be validated through examination. In that regard, the basic perioperative transesophageal echocardiography examination appears to be the natural next step to validate the training of non-cardiac anesthesiologists and intensivists. This type of examination will also be important in other spheres of ultrasonography training, such as FOCCUS.

Anesthesiologists are the critical care physicians of the operating and recovery rooms, and echocardiography (both TEE and TTE) is becoming an integral part of the training curriculum for critical care physicians. Anesthesiologists are rapidly beginning to understand the importance of acquiring these essential skills in resuscitation. Jerath et $a{ }^{1}{ }^{1}$ are adding a powerful tool to the teaching armamentarium, and we congratulate them on such a generous initiative. It will certainly benefit all those interested in this new and rapidly expanding field. This educational module is freely available on the Web (http://pie.med.utoronto.ca/ TEE/index.htm) and is currently being accessed by about 6,000 trainees and medical educators worldwide. Jerath et al. continue to add new teaching modules to their web site, and we look forward to further publications evaluating their educational or training benefit.

We eagerly await the next step.

\section{La formation en échocardiographie transœsophagienne: vivement la prochaine étape!}

Dans ce numéro du Journal, Jerath et coll. ${ }^{1}$ décrivent la façon dont un module interactif d'enseignement de l'échocardiographie transœsophagienne (ÉTO) en ligne doté d'un pré/post-test a considérablement amélioré les performances des stagiaires provenant de plusieurs disciplines. Pourquoi cette initiative est-elle si importante en 2011? Depuis la première description, en 1976, d'une utilisation de sonde d'échocardiographie osophagienne, ${ }^{2}$ des progrès considérables ont été apportés à l'ÉTO ainsi qu'à l'échocardiographie transthoracique (ÉTT) en termes d'équipement, d'indications, d'exigences de formation, d'accréditation et de formation médicale continue. Au fur et à mesure que l'échocardiographie (l'ÉTO puis, à l'avenir, l'ÉTT ciblée) devient de plus en plus une partie intégrante de la formation - non seulement des anesthésiologistes cardiaques, mais aussi des anesthésiologistes généraux et des intensivistes -, certaines questions doivent être abordées.

\section{Indications pour l'utilisation de l'ÉTO}

Comme le décrivent Frazin et coll., ${ }^{2}$ à l'origine l'ÉTO était utilisée chez les patients souffrant de maladie pulmonaire obstructive chronique parce que l'acquisition des images par ÉTT était sous-optimale en raison des modifications subies au niveau de la cavité thoracique. De même, dans la salle d'opération, l'accès au thorax est souvent limité pour des questions de stérilité ou des raisons techniques. C'est pour cette raison que l'échocardiographie transœsophagienne est devenue l'instrument de choix pour le monitorage et le diagnostic de diverses causes d'instabilité hémodynamique en salle d'opération et ce, tout particulièrement en chirurgie cardiaque. Les directives originales de 1996 sur l'utilisation de l'ÉTO ont récemment été mises à jour. ${ }^{3}$ En effet, c'est en 2010 qu'un Groupe de travail conjointement formé par des membres de l'American Society of Anesthesiologists (ASA) et de la Society of Cardiovascular Anesthesiologists, ${ }^{4}$ composé de neuf anesthésiologistes travaillant dans un cadre universitaire ou privé et provenant de différentes régions des États-Unis, a mis à jour ces directives. Le Groupe de travail comptait également deux cardiologues, l'un représentant l'American College of Cardiology et le second l'American Society of Echocardiography, ainsi que deux spécialistes de la méthodologie du Comité des normes et des paramètres de pratique de l'American Society of Anesthesiologists. Leur revue de littérature a porté sur une période de 16 ans allant de 1994 à 2009, et 457 articles au total ont été retenus parmi les 8000 citations initialement envisagées. Des directives ont été rédigées par consensus sur la base de cette analyse. Par exemple, il est recommandé que l'ÉTO soit utilisée pour toutes les interventions aortiques thoraciques et cardiaques. De plus, l'ÉTO devrait être utilisée, lorsqu'elle est disponible, dans les cas où une instabilité hémodynamique inexplicable et potentiellement 
fatale perdure malgré un traitement adapté en chirurgie non cardiaque. En voici quelques exemples: une hypotension ou une hypoxémie persistante inexplicable; la prédiction d'une hypotension potentiellement fatale; la transplantation d'un poumon ou du foie; un traumatisme thoracique ou abdominal majeur; et les interventions aortiques abdominales ouvertes. En outre, il faut également utiliser l'ÉTO aux soins intensifs lorsque l'hypotension ou l'hypoxémie persistante ne peuvent être expliquées. Ces recommandations devraient aboutir à une augmentation considérable de la demande en formation en ÉTO. C'est à cet égard que les travaux de Jerath et coll. ${ }^{1}$ tombent à point nommé.

\section{À qui s'adresse la formation en ÉTO?}

L'échocardiographie transœsophagienne ne devrait être pratiquée que par des personnes ayant reçu une formation adaptée. Les anesthésiologistes cardiaques doivent de plus en plus être accrédités en ÉTO périopératoire par des organismes tels que le National Board of Echocardiography américain (NBE) (www.echoboard.com) afin d'obtenir des privilèges hospitaliers en échocardiographie. Les programmes de stages (les fellowships) en anesthésie cardiaque et les programmes intensifs destinés aux anesthésiologistes déjà en pratique sont actuellement disponibles. Avant 2010, le NBE n'offrait que des programmes d'accréditation avancés. À présent, un nouveau niveau d'accréditation est disponible, l'Échocardiographie transœsophagienne périopératoire de base. Cette accréditation couvre les connaissances de base que les anesthésiologistes non cardiaques et les intensivistes doivent maitriser s'ils souhaitent pratiquer l'ÉTO. Les objectifs des examens de base et avancé du NBE sont décrits dans les tableaux 1 et 2 .

\section{Quelles sont les précautions à prendre avant de réaliser une ÉTO?}

À l'Institut de cardiologie de Montréal, l'examen d'ÉTO est inclus dans le formulaire de consentement général. Sauf en cas de contre-indication, nous réalisons systématiquement un examen cardiaque complet en ÉTO après l'induction de l'anesthésie et l'intubation endotrachéale. La sonde d'ÉTO est maintenue en position jusqu'à la fin de l'intervention. Il est particulièrement important d'insérer la sonde d'ÉTO avant l'administration de l'héparine dans les cas avec circulation extracorporelle (CEC), étant donné que l'insertion de la sonde pendant la CEC a été associée à des saignements gastro-intestinaux. ${ }^{5}$ Les pathologies œsophagiennes ou gastriques prédisposant à la perforation
Tableau 1 Examens et accréditations du National Board of Echocardiography

Objectifs de l'examen d'échographie transœsophagienne périopératoire de base

1. Considérations au niveau de la sécurité du patient

2. Imagerie échocardiographique: acquisition et optimisation

3. Anatomie cardiaque normale et corrélation avec les coupes d'imagerie

4. Fonction ventriculaire globale

5. Fonction systolique ventriculaire régionale et identification des pathologies

6. Identification de base des anomalies des valves cardiaques

7. Identification des masses intracardiaques dans la chirurgie non cardiaque

8. Évaluation de base de l'hémodynamie périopératoire

9. Modalités diagnostiques associées

10. Identification de base des cardiopathies congénitales chez l'adulte 11. Échographie superficielle pour l'accès vasculaire

Tableau 2 Examens et accréditations du National Board of Echocardiography

Objectifs de l'examen d'échographie transœsophagienne périopératoire avancé

1. Les principes de l'échographie

2. Les sondes

3. L'imagerie

4. Les principes de l'échographie Doppler

5. Profils de signaux Doppler pour une physiologie normale et anormale

6. L'échocardiographie quantitative

7. Les artéfacts et les pièges de l'imagerie

8. Matériel, contrôle des infections et sécurité

9. Anatomie et débits normaux pendant l'examen complet

10. Ischémie du myocarde et fonction ventriculaire segmentaire

11. Fonction ventriculaire et l'hémodynamie

12. Identification des contenus intracavitaires

13. Manifestations échocardiographiques des cardiopathies congénitales chez les patients adultes

14. La cardiomyopathie obstructive hypertrophique

15. Les cardiomyopathies dilatées et restreintes

16. L'échocardiographie pour le péricarde et l'anatomie extracardiaque

17. L'échocardiographie pendant la chirurgie cardiaque

18. L'évaluation des valves cardiaques en période périopératoire

19. Les indications de l'échocardiographie transœsophagienne

20. L'imagerie non ÉTO et les autres modalités diagnostiques

ÉTO échocardiographie transœsophagienne (tiré de www.echo board.com)

sont certaines des contre-indications à l'ÉTO, tout comme la détresse respiratoire chez le patient non intubé, l'instabilité de la colonne cervicale, ou une coagulopathie 
grave. Avec l'augmentation de l'utilisation de routine de l'ÉTO hors de la salle d'opération cardiaque, il est crucial d'ajouter des questions liées aux pathologies œsophagiennes au formulaire d'évaluation préopératoire généralement utilisé. Malgré ces précautions, la perforation œsophagienne demeure un risque de l'ÉTO périopératoire. Dans notre centre, sur les 15000 examens d'ÉTO réalisés depuis 1999, trois perforations ont eu lieu (informations tirées de la base de données sur l'ÉTO de l'Institut de cardiologie de Montréal). C'est pourquoi d'autres alternatives doivent être mises à la disposition de l'anesthésiologiste pour les patients à haut risque.

\section{Les alternatives à l'ÉTO}

Dans la salle d'opération cardiaque, l'échocardiographie épicardique peut être utilisée pour remplacer l'ÉTO. Des directives ont été publiées concernant la réalisation d'un examen épicardique. ${ }^{6}$ Dans un contexte non cardiaque ou en salle de réveil, l'échocardiographie au chevet a vu des progrès significatifs chez les patients hémodynamiquement instables après l'extubation trachéale et chez les patients pour lesquels l'ÉTO est contre-indiquée. La combinaison d'un examen physique et d'une échocardiographie au chevet a amélioré le diagnostic clinique et la prise en charge des patients gravement malades. De tels examens échocardiographiques au point d'intervention sont en général 'ciblés' pour répondre à une question clinique spécifique et durent considérablement moins longtemps (moins de six minutes dans certaines études) que l'échocardiographie traditionnelle. ${ }^{7,8}$ L'objectif d'un tel examen n'est pas de réaliser une étude complète et globale, mais plutôt d'améliorer et d'approfondir l'examen physique.

L'échocardiographie transthoracique est une technique non effractive et plus facilement accessible que l'ÉTO; par conséquent, elle devrait être la modalité de choix pour réaliser un examen échocardiographique ciblé. 9 À ce jour, elle n'a pas été beaucoup utilisée dans l'unité des soins intensifs ou la salle d'opération, parce que seuls quelques intensivistes et anesthésiologistes ont reçu une formation en ÉTT. ${ }^{10}$ Cela étant, au cours des dernières années, on a assisté à une augmentation du nombre d'échocardiographies au chevet réalisées par des non-cardiologues, des intensivistes et des anesthésiologistes dans le but d'obtenir des informations diagnostiques qui ne peuvent être évaluées par un examen physique seul. ${ }^{1-13}$ L'examen d'ÉTT, baptisé FOCUS (FOcused Cardiac Ultrasound Study) ou FOCCUS (FOcused Critical Care Ultrasound Study), se définit comme une ÉTT réalisée avec des objectifs limités spécifiques. ${ }^{10}$ Parmi les objectifs FOCUS, citons l'évaluation de la fonction ventriculaire gauche et droite, l'évaluation de l'espace péricardique pour l'épanchement et la tamponnade, et l'évaluation de l'état de volume. Les objectifs FOCCUS supplémentaires comprennent l'évaluation des pathologies pulmonaires, des liquides dans l'abdomen, des vaisseaux, etc. La compétence en ÉTT devra en fin de compte être établie selon des niveaux prédéterminés de formation (par ex., niveaux 1 à 4). Certaines directives de formation ont déjà été proposées, ${ }^{14-16}$ notamment un consensus entre l'American College of Chest Physicians et la Société de Réanimation de Langue Française. ${ }^{17}$

\section{L'apprentissage de l'échocardiographie: approches standard et approches avancées}

L'apprentissage de l'échocardiographie comprend des lectures dirigées et des conférences, de la pratique supervisée en peropératoire ou à l'unité des soins intensifs, et des stages en échocardiographie. Dans ce numéro du Journal, Jerath et coll. ${ }^{1}$ présentent leur expérience quant à la mise au point d'un programme en ligne qui permet aux utilisateurs de visualiser 20 coupes diagnostiques standard d'ÉTO en conjonction avec un modèle tridimensionnel du cœur (3D). Afin de valider la valeur éducationnelle de ce matériel d'enseignement, les auteurs ont analysé la performance des stagiaires après qu'ils ont eu accès au matériel de formation pendant une moyenne de $130 \mathrm{~min}$. Les auteurs ont observé une augmentation significative de $31 \%$ dans les notes de test des stagiaires. Les stagiaires ont également évalué ce programme comme étant très utile, lui accordant une note de 4,7 sur 5. De plus, Jerath et coll. ${ }^{1}$ ont démontré qu'il était possible, grâce à leur test, de faire la distinction entre utilisateurs novices ou experts de l'ÉTO. Ce site Internet est vivement recommandé pour les personnes qui suivent une formation en ÉTO, et il s'agit d'une contribution majeure à la formation par le Dr Jerath et le groupe d'anesthésiologistes de Toronto. De plus, de telles initiatives ouvrent la voie à l'identification de pathologies et à l'apprentissage de l'ÉTT. En réalité, les images d'ÉTT offrent simplement une perspective différente des images d'ÉTO (voir figure). Par extension, cette initiative pourrait aussi être adaptée pour l'enseignement de l'échographie cardiothoracique, de l'abdomen et des autres grands vaisseaux du corps. En outre, de tels outils éducationnels sont un complément idéal aux simulateurs. L'avantage majeur de la simulation est que l'étudiant en échocardiographie peut être exposé à des pathologies rares telles que la dissection aortique et la tamponnade cardiaque. Le besoin de ressources supplémentaires pour l'enseignement de cette compétence est bien réel. Ce site Internet (http://pie.med.utoronto. ca/TEE/index.htm) offre aujourd'hui un outil 
d'enseignement validé qui est accessible gratuitement à tous. Mais cette formation devra, en fin de compte, être validée par un examen. À cet égard, l'examen d'échocardiographie transœsophagienne périopératoire de base semble être la voie naturelle à suivre si l'on souhaite valider la formation des anesthésiologistes non cardiaques et des intensivistes. Ce type d'examen sera également important dans d'autres domaines de la formation en échographie, notamment dans l'examen FOCCUS.

Les anesthésiologistes sont les intensivistes des salles d'opération et de réveil, et l'échocardiographie (ÉTO et ÉTT) est sur le point de devenir une partie intégrante du programme de formation des intensivistes. Les anesthésiologistes sont en train de réaliser rapidement l'importance d'acquérir ces compétences essentielles en matière de réanimation «échoguidée». Jerath et coll. ${ }^{1}$ ajoutent ici un outil puissant à l'arsenal éducationnel, et nous tenons à les féliciter de leur généreuse initiative. Il ne fait aucun doute que cet outil profitera à toutes les personnes qui s'intéressent à ce domaine nouveau et croissant rapidement. Ce module de formation est disponible gratuitement sur Internet (http://pie.med. utoronto.ca/TEE/index.htm); à l'heure actuelle, quelques 6000 stagiaires et formateurs en médecine y accèdent depuis les quatre coins de la planète. Jerath et coll. continuent d'ajouter de nouveaux modules d'enseignement à leur site Internet, et nous nous réjouissons des publications futures qui évalueront leurs avantages éducationnels.

Vivement la prochaine étape!

Conflict of interest Dr. Denault is cosultant for Pfizer.

\section{References}

1. Jerath A, Vegas A, Meineri $M$, et al. An interactive online 3D model of the heart assists in learning standard transesophageal echocardiography views. Can J Anesth 2011; 58. DOI: 10.1007/s12630-010-9410-5.

2. Frazin L, Talano JV, Stephanides L, Loeb HS, Kopel L, Gunnar $R M$. Esophageal echocardiography. Circulation 1976; 54: 102-8.

3. Anonymous. Practice guidelines for perioperative transesophageal echocardiography. A report by the American Society of Anesthesiologists and the Society of Cardiovascular Anesthesiologists
Task Force on Transesophageal Echocardiography. Anesthesiology 1996; 84: 986-1006.

4. American Society of Anesthesiologists and the Society of Cardiovascular Anesthesiologists Task Force on Transesophageal Echocardiography. Practice guidelines for perioperative transesophageal echocardiography. An updated report by the American Society of Anesthesiologists and the Society of Cardiovascular Anesthesiologists Task Force on Transesophageal Echocardiography. Anesthesiology 2010; 112: 1084-96.

5. St Pierre J, Fortier LP, Couture P, Hebert Y. Massive gastrointestinal hemorrhage after transoesophageal echocardiography probe insertion. Can J Anaesth 1998; 45: 1196-9.

6. Reeves ST, Glas KE, Eltzschig H, et al. Guidelines for performing a comprehensive epicardial echocardiography examination: recommendations of the American Society of Echocardiography and the Society of Cardiovascular Anesthesiologists. J Am Soc Echocardiogr 2007; 20: 427-37.

7. Rugolotto M, Chang CP, Hu B, Schnittger I, Liang DH. Clinical use of cardiac ultrasound performed with a hand-carried device in patients admitted for acute cardiac care. Am J Cardiol 2002; 90: 1040-2.

8. Gorcsan J 3rd, Pandey P, Sade LE. Influence of hand-carried ultrasound on bedside patient treatment decisions for consultative cardiology. J Am Soc Echocardiogr 2004; 17: 50-5.

9. Joseph MX, Disney PJ, Da Costa R, Hutchison SJ. Transthoracic echocardiography to identify or exclude cardiac cause of shock. Chest 2004; 126: 1592-7.

10. Manasia AR, Nagaraj HM, Kodali RB, et al. Feasibility and potential clinical utility of goal-directed transthoracic echocardiography performed by noncardiologist intensivists using a small hand-carried device (SonoHeart) in critically ill patients. J Cardiothorac Vasc Anesth 2005; 19: 155-9.

11. Beaulieu Y, Marik PE. Bedside ultrasonography in the ICU: part 1. Chest 2005; 128: 881-95.

12. Beaulieu $Y$, Marik PE. Bedside ultrasonography in the ICU: part 2. Chest 2005; 128: 1766-81.

13. Beaulieu $Y$. Bedside echocardiography in the assessment of the critically ill. Crit Care Med 2007; 35: S235-49.

14. Langlois SL. Focused ultrasound training for clinicians. Crit Care Med 2007; 35: S138-43.

15. Mazraeshahi RM, Farmer JC, Porembka DT. A suggested curriculum in echocardiography for critical care physicians. Crit Care Med 2007; 35: S431-3.

16. Price $S$, Via $G$, Sloth E, et al. Echocardiography practice, training, accreditation in the intensive care: document for the World Interactive Network Focused on Critical Ultrasound (WINFOCUS). Cardiovasc Ultrasound 2008; 6: 49.

17. Mayo PH, Beaulieu Y, Doelken P, et al. American College of Chest Physicians/La Société de Réanimation de Langue Francaise statement on competence in critical care ultrasonography. Chest 2009; 135: 1050-60. 